\title{
Multi Objective Segmentation for Vehicle License Plate Detection with Immune-based Classifier: A General Framework
}

\author{
Musab Mohammed \\ Bagabir \\ College of Computer \\ Science and Information \\ Technology, \\ Sudan University for \\ Science and echnology, \\ Khartoum, Sudan
}

\author{
Siti Mariyam \\ Shamsuddin, \\ UTM Big Data Centre, \\ Universiti Teknologi \\ Malaysia, \\ Johor, Malaysia
}

\author{
Mohammed Elhafiz \\ College of Computer \\ Science and Information \\ Technology, \\ Sudan University for \\ Science and \\ Technology,
}

\author{
Ali Ahmed \\ Faculty of Engineering, \\ Karay Universit, \\ Khartoum, Sudan
}

\begin{abstract}
Vehicle License Plate Recognition (VLPR) is an important system for harmonious traffic. Moreover this system is helpful in many fields and places as private and public entrances, parking lots, border control and theft control. This paper presents a new framework for Sudanese VLPR system. The proposed framework uses Multi Objective Particle Swarm Optimization (MOPSO) and Connected Component Analysis (CCA) to extract the license plate. Horizontal and vertical projection will be used for character segmentation and the final recognition stage is based on the Artificial Immune System (AIS). A new dataset that contains samples for the current shape of Sudanese license plates will be used for training and testing the proposes framework.
\end{abstract}

Keywords: Multi Objective Particle Swarm Optimization; Artificial Immune System; Vehicle License Plate Recognition; Connected Component Analysis

\section{INTRODUCTION}

Therefore, many control and surveillance applications have been used VLPR Vehicle license plate recognition (VLPR) is an important component for automating many control and surveillance systems, such as road traffic monitoring, parking lots, access control, highway electronic toll collection, red light violation enforcement, finding stolen cars, gathering traffic flow statistics [1].

For the last few decades and due to the difference of license plates in formats, styles, colors and size from country to others the field of VLPR and its application has attracted many researchers in many countries to search and develop systems in order to identify their own vehicles license plate numbers. So far, many methods have been proposed for VLPR depending on the country's license plate characteristics.

VLPR is a technique that involves image processing technology and computer vision. Recognition/identification algorithms are generally composed of four major parts; preprocessing, license plate localization/detection, character segmentation and character recognition, and each part may contain several steps.

A considerable amount of literature has been published on VLPR, some of the related work is as follows: Saqib Rasheed, Asad Naeem and Omer Ishaq [2] proposed a technique of automated number plate recognition, which using canny edge detection operator and Hough lines for license plate localization, and template matching in recognition part.

In the recognition system proposed by Alginahi [3] for Saudi Arabian's license plate, in pre-processing, the median filter is used to remove noise, and then the sobel detector is applied.
As a next step, the license plate is detected by searching the vertical lines, then the width to length ratio is calculated if more than two vertical are detected. Character segmentation is then performed by using the 8-connected components technique to find all the components (characters/numerals) on the plate. Then the horizontal projection profiles and zoning is used as features extractor. And finally to recognize the plate both a Mahalanobis distance classifier and a Multilayer Perceptron Neural Network classifier are used.

In [4] the Saudi Arabian's license plate detection depends on a black cross that centers the plate, so an edge detector is applied to find the horizontal and vertical maps, then before median filter performed, the binary image is obtained by using the average value of pixels in each map as a threshold. After the numbers and letters were segmented the recognition part is performed using a template matching.

In [5] Radial Basis Function (RBF) neural network was used both for the detection and recognition of Libyan's license plate. The pre-possessing phase starts with obtain the binary scale image, performing edge detection using sobel's mask operator, performing dilation process, using 'flood fill' algorithm to fill the interior gaps, applying the filtering task, and finally the image was smoothened by eroding it twice. The character segmentation phase is based on thresholding and CCA. A matrix of size 4 x 2 contains a character feature, which is obtained by dividing a character image into a sequence of horizontal "scan lines" by using the raster scanning.

In [6] the authors used Canny edge detection operator to locate the license plate in the image, the binary large object (blob) coloring algorithm is used in character segmentation part, the feature vector of a character image was encoded by 
using Average Absolute Deviation algorithm, in the classification phase a multi layered perceptron artificial neural network model was used, in addition to, the numbers and the letters were classified by using two separate ANN.

According to image segmentation and the multi-level thresholding problem in image segmentation, many techniques are proposed using Particle Swarm Optimization (PSO) Algorithm such as in [7,9].

In [7] an approach on the basis of the PSO with wavelet mutation is used. The optimization of the multi-level thresholds for the images is performed by maximizing the total entropy of the image. In [8] the algorithm is used PSO to maximize the entropy criterion (Kapur's) and between-class variance (Otsu's) objective functions.[9] presents a hybrid of PSO and Genetic algorithm for multi-level thresholding base on maximum entropy criterion .

Other techniques are used to solve the multi-level thresholding problem in image segmentation as Genetic Algorithm in [10], Hill Climbing in [11], and Artificial Bee Colony (ABC) Algorithm in [12] which approximates the 1-D histogram of an image by calculating the Gaussian mixture model parameters.

In the recognition and classification phase, AIS is the quite popular and active research topic in recognition domain. As [13] and [14] describe the implementation of AISs in solving an image classification problem. In [13] the clonal selection algorithm proposed in a shape recognition problem beside hamming distance to calculate the affinity. In [14] the authors proposed a handwritten character recognition algorithm based on AIS by using clone selection principle .

While this study will focus on Sudanese vehicles license plates, each country has its own rule on vehicle license plate designing. The following section will give some important information about the type, shape and design of Sudanese vehicles license plates.

The paper is organized as follows. The next section constitutes an introduction about the Sudanese vehicle license. Section III introduces the Multi-objective optimization and Multi-objective Particle Swarm optimization methods .The complete framework is presented in details in Section IV. Section $\mathrm{V}$ present a discussion, the conclusion is presented in Section VI.

\section{SUDANESE VEHICLE LICENSE}

\section{PLATE}

The Sudanese vehicles license plates are categorized in a number of types, that categorization was based on the differences of plates background color and characters color, some of types are mentioned in Table 1 below:

Table 1. Sudanese Vehicles Plates Types

\begin{tabular}{|c|c|c|}
\hline Type & $\begin{array}{c}\text { Background } \\
\text { Color }\end{array}$ & $\begin{array}{c}\text { Characters } \\
\text { Color }\end{array}$ \\
\hline Private vehicles & White & Black \\
\hline $\begin{array}{c}\text { Commercials } \\
\text { (Passenger) }\end{array}$ & White & Green \\
\hline Commercials & Black & White \\
\hline
\end{tabular}

www.ijcat.com

\begin{tabular}{|c|c|c|}
\hline (Goods) & & \\
\hline Police & Blue & White \\
\hline Government & Yellow & Black \\
\hline
\end{tabular}

The size of all plate types is $32 \times 16$ centimetres (See Figure1). The plate has been divided into three regions; one region in the upper part, that contains the name of the country 'SUDAN' written in English and Arabic. The other two regions in the lower part, which divided by a silver metallic bar, one on the right this region contains numerals ( 1 to 5 numbers) written in English and Arabic, and the other region on the left side contains characters or character and number written in English and Arabic, the characters are an abbreviation of Sudan states' names, and the number to keep the sequence of the numbering. This study will focus on the first type: private vehicles as show in Figure 1.

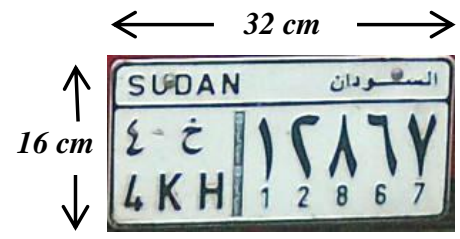

Figure 1. Sudanese License Plate

\section{MULTI OBJECTIVE PARTICLE SWARM OPTIMIZATION}

Multi Objective Optimization (MOO) also known as multi criterion extends the optimization theory by permitting several design objectives to be optimized simultaneously, the goal is to find a set of values for the design variables that simultaneously optimizes several objective (or cost) functions [16]. In mathematical terms, A MOO problem can be formulated as :

Minimize $\vec{f}(\vec{x}):=\left[f_{1}(\vec{x}), f_{2}(\vec{x}), \ldots f_{k}(\vec{x})\right]$

Subject to the constraints

$$
\begin{array}{ll}
g_{i}(\vec{x}) \leq \mathrm{O}, & \mathrm{i}=1,2, \ldots, \mathrm{m} \\
\boldsymbol{h}_{j}(\vec{x})=\mathrm{O}, & \mathrm{j}=1,2, \ldots, \mathrm{p}
\end{array}
$$

where $g_{i}(\vec{x})=\left[x_{1}, x_{2}, \ldots, x_{n}\right]^{T}$ is the vector of design variables, $f_{i}: \mathfrak{R}^{n} \rightarrow \mathfrak{R}, \mathrm{i}=1, \ldots, \mathrm{k}$ are the objective functions and $g_{i}, h_{j}: \mathfrak{R}^{n} \rightarrow \mathfrak{R}, \mathrm{i}=1, \ldots, \mathrm{m}, \mathrm{j}=$ $1, \ldots, \mathrm{p}$ are the constraint functions of the problem. The objectives (2) and (3) often conflict with each other. Improvement of one objective may lead to aggravation of another. Thus, a single solution which can optimize all objective simultaneously does not exist. As an alternative, the best trade off solutions is called the Pareto Optimal Solution.

There are different approaches to solving MOO problems such as aggregating, population based non-pareto and paretobased techniques [20]. A comprehensive review of the 
different approaches to solving MOO problems can be found in [20].

Particle Swarm Optimization (PSO) is developed by Kenney and Eberhart in 1995 [17], it's a population-based metaheuristic inspired on the social behavior of birds within a flock. In a PSO algorithm each potential solution to the problem is called a particle and the population of solutions is called a swarm. The way in which PSO updates the particle $x_{i}$ at the generation $t$ is through the formula[22]:

$x_{i}(t)=x_{i}(t-1)+v_{i}(t)$

where the factor $v_{i}(t)$ is known as velocity and it is given by

$v_{i}(t)=w^{*} v_{i}(t-1)+C 1 * r 1 *\left(x_{\text {pbest }_{i}}-x_{i}\right)+$

$C 2 * r 2 *\left(x_{\text {gbest }_{i}}-x_{i}\right)$

In this formula, $x_{\text {pbest }_{i}}$ is the best solution that $\mathrm{x} \mathrm{i}$ has viewed, $x_{\text {gbest }_{i}}$ is the best particle (also known as the leader) that the entire swarm has viewed, $w$ is the inertia weight of the particle and controls the trade-off between global and local experience, $\mathrm{r} 1$ and $\mathrm{r} 2$ are two random numbers within the interval $[0,1]$, and $\mathrm{C} 1$ and $\mathrm{C} 2$ are specific parameters which control the effect of the pbest and gbest particles.

The simplicity of PSO have made it a popular optimization approach, and a good candidate to be extended for multi objective optimization (MOO) [21]. This technique called Multi Objective Particle Swarm Optimization (MOPSO) has been introduced in [21] to deal with MOO problems. More details about MOPSO can be found in [20,21]

Image segmentation based on thresholding techniques can be seen as a multi objective problem. Where, there is no single threshold objective function (criterion) able to produce an optimal thresholds values for all kinds of images [15, 23]. The use of MOPSO aims to obtain a good segmentation results on more kinds of images. Consequently, the segmentation problem can be formulated mathematically as:

Minimize/Maximize

$$
f_{m}\left(t_{1}, \ldots, t_{N-1}\right) \quad \mathrm{m}=1,2, \ldots, \mathrm{M}
$$

Subject to

$$
0<t_{1}<t_{2}<\ldots<t_{N-1}<L
$$

Where $M$ is the number of objective functions (criterion) used for the segmentation, $t_{i}$ the segmentation thresholds, and $\mathrm{L}$ the number of gray levels.

In our work, two broadly used optimal thresholding methods namely entropy criterion [19] method and betweenclass variance [18] method will be used as our objective functions.

\section{THE FRAMEWORK}

The proposed framework is designed for Sudanese vehicle license plate recognition. This system is composed of a number of stages as shown in figure 2 are pre-processing that includes the MOPSO, Plate Detection/Localization, Character Segmentation and AIS Recognizer/Classifier.

The input of the system is the original image of the vehicle in RGB scale of size $769 \times 559$ pixels taken from different distances and Angles. The details of other stages are presented in the following subsections.

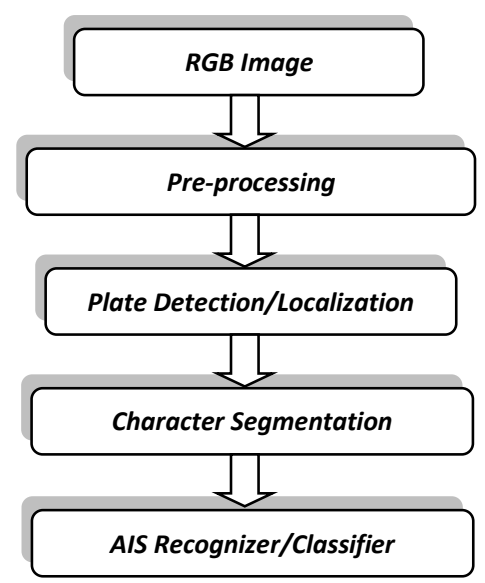

Figure 2. The Proposed Framework Diagram

\subsection{Pre-processing}

Pre-processing stage plays an important role, which influences the accuracy of the plate detection stages significantly. In this study the first step in pre-processing is the RGB image conversion into gray scale, as the gray scale decreases the computational time; the RGB image consists of three channels red, green and blue, the value of each channel in the range 0 256 , whereas the gray scale image contains only one channel. Then, in this framework we apply median filtter to remove noises like random occurrences of black and white pixels, then the image contrast will be adjusted by using histogram equalization technique.

Image segmentation is a process of partitioning a digital image into multiple segments, so it aims to partitioning an image into regions in order that each region groups adjacent pixels having similar attributes as intensity. Multi-level thresholding is one of the most important image segmentation techniques, which decrease the number of intensity levels. The basic idea of multi-level thresholding technique is to divide the pixels into several groups based on a certain number of threshold values. The reason behind the adoption of the multi-level thresholding is to clearly separate the plate region in the image from the background. Therefore, the automatic selection of optimal thresholds has remained a challenge in image segmentation [15].

In this framework, we propose a new image segmentation technique based on multi objective particle swarm optimization MOPSO, that combines the flexibility of multi objective fitness functions with the power of PSO for searching vast combinatorial state spaces, in order to find the optimal thresholds.

\subsection{Plate Detection/Localization}

The aim of this stage is to extract the license plate from the gray scale image. Therefore the proposed framework provides more importance to this stage whose success guarantees the successful performance of the VLPR system. 
As a first step in this stage, the gray scale image resulted by the previous stage will be converted to binary image using the well-known Otsu global thresholding method [18]. Other Other morphological operations may also be used in order to isolate the plate from the background.

The resultant binary image will be labeled (Connected Components Labeling-CCA) in order to identify objects/regions in the binary image (each region of connected pixels is called connected component). Then CCA will be used to analyze the regions independently and measure the property of each region. Since the Sudanese license plate have a unique features such as the following: rectangular shape, the ratio of the width to height of the rectangle is fix and the area of the rectangle, this features will be useful to identify the plate region.

\subsection{Character Segmentation}

Character segmentation is the procedure of extracting and isolating the characters and numbers (each character and number) on the license plate image. At this phase, the English characters and Arabic numbers would be considered as output target in this phase. The study will focus on segmenting the English characters and Arabic numbers as shown in figure 3.

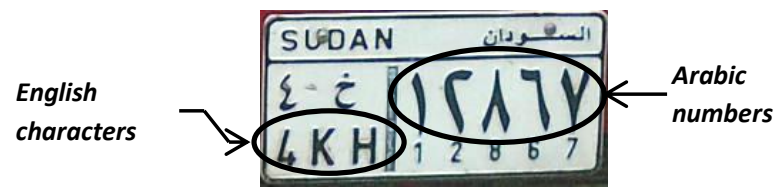

Figure 3. English characters and Arabic numbers with in Sudanese License Plate

At the beginning of character extraction phase and for accurate character segmentation first need is to remove skew from the extracted license plate image. The whole idea of skew correction depends on finding the angle of rotation, and then the extracted license plate image is rotated using that angle of rotation. Then some enhancement techniques will be used as well as morphological operations in order to overcome noise and light variance problems on extracted license plate image.

Then the horizontal projection and vertical projection techniques were proposed to perform the character segmentation process [3][4]. First removing the upper part that containing the words 'SUDAN السودان', then dividing the plate into two images; character on left part(left image) and numbers on right part(right image). Then each image is segmented into isolated characters and numbers after removing the lower part form the right image and upper part from the left image. After this phase all the identification contents of the license plate were prepared for the recognition phase.

\subsection{AIS Recognizer/Classifier}

The final phase in the license plate recognition process is character recognition. Although there are many techniques presented and applied for character recognition, in this work, character recognition is performed based on AIS [14].
First, all segmented characters images will be normalized and resized to a fixed length and width. The character recognition will carried out using Clonal Selection Algorithm [13].

In our proposed AIS the segmented characters and templates of English character and Arabic numbers will considered as antigens and antibodies respectively. The affinity will be calculated using Hamming Distance.

\section{DISCUSSION}

The data set that will be used in the experiments contains 200 color images with a size of $640 \times 480$ pixel, acquired from Sudanese Traffic Police. The tested images have been acquired from the front of vehicles under various illumination and weather conditions (sunny, cloudy, daytime, nigh time, rainy days...etc).

The recognition system receives the image of the target vehicle in RGB format, the meadian filter will be used after the image is converted into gray scale to remove noise.

By performing MOPSO technique on the gray scale image the plate region will be clearly separated from the background, then before applying morphological operations the image is converted to black and white image.

The plate region in the resultant image is determined by applying CCA on the binary image, and the outputs of this process are rectangular shaped regions. In order to identify the plate region, certain features, which are unique to plate, such as mentioned earlier will be used. The final output of plate detection phase is the license plate coordinates, which are used to crop the license plate from the original gray image.

The character segmentation phase receives the extracted vehicle plate image as input. A pre-processing step is required to improve and enhance the plate image, such as skew correction and noise removal. Then horizontal projection and vertical projection techniques are used for segmentation and extraction process. The outputs of this phase are images of each English character and each Arabic number within the license plate.

After the individual characters are extracted, these extracted characters will be passed to the AIS as antigens for classification and recognition task.

\section{CONCLUSION}

The purpose of this paper is to presents a framework for an automatic vehicle license plate recognition system. It describes the proposed framework that will be use to recognize the Arabic numbers and English characters within the Sudanese private vehicle license plates. According to the state of the art of VLPR system a suitable collection of that techniques and methods has been chosen to implement the relevant part of this the proposed framework: MOPSO and CCA are chosen for plate detection and extraction; horizontal and vertical projection are found suitable for segmentation the plate character; AIS for character recognition. The proposed framework is expected to be able to succeed in recognizing the plates efficiently and accurately.

\section{REFERENCES}

[1] Hu, H., Zhang, Z., \& Bai, Y. (2012). Car License Plate Location Based on Mathematical Morphology. In Recent Advances in Computer Science and Information Engineering . Springer Berlin Heidelberg: 415-420. 
[2] Rasheed, S., Naeem, A., \& Ishaq, O. (2012). Automated Number Plate Recognition using hough lines and template matching. In Proceedings of the World Congress on Engineering and Computer Science Vol. 1: 24-26.

[3] Alginahi, Y. M. (2011). Automatic arabic license plate recognition. International Journal of Computer and Electrical Engineering 3.3: 454-460.

[4] Basalamah, S. (2013). Saudi License Plate Recognition. International Journal of Computer \& Electrical Engineering 5.1.

[5] Abulgasem, Nureddin A., Dzulkifli Mohamad, and Siti Zaiton Mohamad Hashim. (2011). Automatic License Plate Detection and Recognition Using Radial Basis Function Neural Network. International Journal of Computer Vision and Applications (IJCVA) 1.1.

[6] Erdinc Kocer, H., \& Kursat Cevik, K. (2011). Artificial neural networks based vehicle license plate recognition. Procedia Computer Science 3: 1033-1037.

[7] Jiang, F., Frater, M. R., \& Pickering, M. (2012). Threshold-based image segmentation through an improved particle swarm optimisation. In Proceedings of the International Conference on Digital Image Computing Techniques and Applications (DICTA):1-5. IEEE.

[8] Duraisamy, S. P., \& Kayalvizhi, R. (2010). A new multilevel thresholding method using swarm intelligence algorithm for image segmentation. Journal of Intelligent Learning Systems and Applications 2.3: 126.

[9] Baniani, E. A., \& Chalechale, A. (2013). Hybrid PSO and Genetic Algorithm for Multilevel Maximum Entropy Criterion Threshold Selection. International Journal of Hybrid Information Technology 6.5.

[10] Banimelhem, O., \& Yahya, Y. A. (2011). MultiThresholding Image Segmentation Using Genetic Algorithm. In World Congress in Computer Science, Computer Engineering, and Applied Computing.

[11] Nath, S., Agarwal, S., \& Kazmi, Q. A. (2011). Image histogram segmentation by multi-level thresholding using Hill climbing algorithm. International Journal of Computer Applications 35.1.

[12] Cuevas, E., Sención, F., Zaldivar, D., Pérez-Cisneros, M., \& Sossa, H. (2012). A multi-threshold segmentation approach based on Artificial Bee Colony optimization. Applied Intelligence 37.3: 321-336.
[13] Isa, N., Sabri, N. M., Jazahanim, K. S., \& Taylor, N. K. (2010). Application of the Clonal Selection Algorithm in artificial immune systems for shape recognition. In Proceedings of the International Conference on Information Retrieval \& Knowledge Management,(CAMP) : 223-228. IEEE.

[14] Chen, Y., Liang, C., Yang, D., Peng, L., \& Zhong, X. (2010). A handwritten character recognition algorithm based on artificial immune. In Proceedings of the International Conference on Computer Application and System Modeling (ICCASM) Vol. 12 : V12-273. IEEE.

[15] Duraisamy, S. P., \& Kayalvizhi, R. (2010). A new multilevel thresholding method using swarm intelligence algorithm for image segmentation. Journal of Intelligent Learning Systems and Applications 2.3: 126.

[16] Nakib, A., Oulhadj, H., \& Siarry, P. (2010). Image thresholding based on Pareto multiobjective optimization. Engineering Applications of Artificial Intelligence $23.3: 313-320$.

[17] James, K., \& Russell, E. (1995). Particle swarm optimization. In Proceedings of International Conference on Neural Networks :1942-1948. IEEE.

[18] Otsu, N. (1975). A threshold selection method from gray-level histograms.Automatica, 11(285-296): 23-27.

[19] Kapur, J. N., Sahoo, P. K., \& Wong, A. K. (1985). A new method for gray-level picture thresholding using the entropy of the histogram. Computer vision, graphics, and image processing $29.3: 273-285$.

[20] Reyes-Sierra, M., \& Coello, C. C. (2006). Multiobjective particle swarm optimizers: A survey of the state-of-the-art. International journal of computational intelligence research $2.3: 287-308$.

[21] Coello, C. A. C. (2011). An introduction to multiobjective particle swarm optimizers. In Soft Computing in Industrial Applications : 3-12 . Springer Berlin Heidelberg.

[22] Durillo, J. J., García-Nieto, J., Nebro, A. J., Coello, C. A. C., Luna, F., \& Alba, E. (2009). Multi-objective particle swarm optimizers: An experimental comparison. In Evolutionary Multi-Criterion Optimization : 495-509 . Springer Berlin Heidelberg. 